\title{
Comparison of protocol based cancer therapies and discrete controller based treatments in the case of endostatin administration
}

\author{
Johanna Sápi*, Dániel András Drexler**, Levente Kovács* \\ *Research and Innovation Center of Óbuda University, Physiological Controls Group, \\ Óbuda University, Budapest, Hungary \\ Email: \{sapi.johanna, kovacs.levente\}@nik.uni-obuda.hu \\ ** Department of Control Engineering and Information Technology, \\ Budapest University of Technology and Economics, Budapest, Hungary \\ Email: drexler@iit.bme.hu
}

\begin{abstract}
In the medical practice, there are several methods to administer anti-cancer drugs. A commonly used method is the intermittent bolus doses (BD) administration when the patient receives drug on given days and the therapy has rest periods between the injections. The amount of bolus doses can be the maximum tolerated dose (MTD) or less. Anti-cancer drug can be administered in low doses over prolonged periods without extended rest periods which is called as low-dose metronomic therapy (LDM). In addition, continuous infusion therapy is applicable within clinical environment, not yet as a portable device. The major disadvantage of these methods is the empiricism associated with determining the optimal biologic dose (OBD). In order to solve the problem, we have designed discrete-time controllers which realize automated optimal treatments.

Keywords - cancer therapy, protocol, discrete-time controller, endostatin, bolus doses, maximum tolerated dose, low-dose metronomic therapy, state feedback, setpoint control, actual state observer, load estimation
\end{abstract}

\section{INTRODUCTION}

Newest research studies on cancer treatments show the importance of specific cancer therapies. The group of Targeted Molecular Therapies (TMTs) [1, 2] contains several different therapeutic methods; however what they have in common is the aim to fight directly against specific, identified cancer mechanisms. Antiangiogenic therapy [3] is a type of TMT, which acts by inhibiting tumor vascularization. Ceasing the process of angiogenesis (new blood vessel formation), tumor growth is limited.

There are several types of antiangiogenic drugs (also known as angiogenic inhibitors) like bevacizumab [4], angiostatin [5] and endostatin [6]. Delivery of an antiangiogenic drug is not a trivial problem since the biological effectiveness of the treatment highly depends on the applied dosage. Using an empirical method to achieve the biological effective dosage, it is not guaranteed that the optimal therapy will be found; however, using closed-loop control, optimal solution can be realized $[7,8]$.

In this paper, we discuss the possible delivery methods in cancer therapies which use endostatin. Endostatin is able to target neovascular endothelial cells (ECs) and has the potential for antiangiogenic and antitumor activities
[9]. However, it has to be taken into account that endostatin has a short half-time [10].

The paper is organized as follows. In Section II, we present the nonlinear model of tumor growth under angiogenic inhibition. Section III discusses first the cancer protocols in the light of the dosage problem, and then it presents the simulation results of the protocol based cancer therapies. In Section IV, first the design aspects of the discrete time controller with state feedback, setpoint control, actual state observer and load estimation are summarized, and then the simulation results of the discrete-time controller based treatments are evaluated. The paper ends with the results and discussion in Section V.

\section{THE APPLIED MODEL OF TUMOR GROWTH}

P. Hahnfeldt et al. created a dynamic model for tumor growth under antiangiogenic therapy [11]. In a simplified version of this model, continuous infusion therapy is taken into account [12], where the input (the inhibitor administration rate) is equal to the concentration of administered inhibitor (serum level of inhibitor) and the system is described by the equations:

$$
\begin{aligned}
& \dot{x}_{1}=-\lambda_{1} x_{1} \ln \left(\frac{x_{1}}{x_{2}}\right) \\
& \dot{x}_{2}=b x_{1}-d x_{1}{ }^{2 / 3} x_{2}-e x_{2} g \\
& y=x_{1},
\end{aligned}
$$

where $x_{1}$ is the tumor volume $\left(\mathrm{mm}^{3}\right), x_{2}$ is the endothelial/ vascular volume $\left(\mathrm{mm}^{3}\right)$ and $g$ is the concentration of the administered inhibitor $(\mathrm{mg} / \mathrm{kg})$. The model contains the following parameters: $\lambda_{l}$ is the tumor growth rate (1/day), $b$ is the stimulatory capacity of the tumor to the vasculature (1/day), $d$ is the endogenous inhibition of previously generated vasculature $\left(1 /\left(\right.\right.$ day $\left.\left.\cdot \mathrm{mm}^{2}\right)\right), e$ is the antiangiogenic effect of the administered inhibitor on the tumor vasculature $(\mathrm{kg} /($ day $\cdot \mathrm{mg}))$.

Parameter values for the considered Lewis lung carcinoma and the mice used in the experiment are [11]: $\lambda_{I}=0.1921 /$ day, $b=5.851 /$ day, $d=0.008731 /$ day $\cdot \mathrm{mm}^{2}$. The experiment has shown that the most effective inhibitor was endostatin; therefore, we have applied this 
antiangiogenic drug in controller design (the value of parameter $e$ for endostatin is $e_{\text {endostatin }}=0.66 \mathrm{~kg} /($ day $\left.\cdot \mathrm{mg})\right)$.

\section{PROTOCOL BASED CANCER THERAPIES}

\section{A. Cancer protocols in the light of the dosage problem}

Methods of drug administration fall into four categories: a) bolus doses (BD), b) bolus doses with maximum tolerated dose (BD MTD), c) low-dose metronomic (LDM) regimen, and d) continuous infusion therapy. In this subsection, we draw a distinction between these treatments; in addition, we lay emphasis on the possible delivery doses in the case of endostatin treatment.

Using intermittent bolus doses administration, the patient receives drug on given days and the therapy has rest periods between the injections. The injected amount of boluses can be the maximum tolerated dose (MTD) or lower dose. The length of the rest periods depends on the amount of boluses (e.g. after MTD a longer rest period is required). The disadvantage of this method is that it involves regrowth of tumor cells, and in several cases the growth of selected clones will be resistant to the therapy [13].

To avoid the adverse events of bolus doses, anti-cancer drug can be administered in low doses over prolonged periods without extended rest periods which is called as low-dose metronomic therapy [14]. Advantages of LDM are its antitumor efficacy and reduced acute toxicities; however the major disadvantage is the empiricism associated with determining the optimal biologic dose (OBD) [15]. This is the most important problem oncologists are faced with when they are trying to translate LDM into the clinical application; however, according to our previous research, this can be solved by a closed-loop control [16].

Low-dose daily administration of antiangiogenic drugs was found to be more effective than high-doses with rest periods. In [16], the effect of low-dose bevacizumab treatment on mice and human adenocarcinoma was examined. Article [17] makes a case for low-dose endostatin treatment on human lung cancer. In this experiment, $3 \mathrm{mg} / \mathrm{kg}$ endostatin was administered intravenously daily for 10 days. The results lend support to the view that endostatin normalizes the structure and function of tumor vasculature. The importance of tumor vasculature normalization lies on the further delivery of chemotherapeutic drugs. Low-dose antiangiogenic therapy creates the possibility of further efficient therapeutic agent use (if any) or assists the more effective direct antitumor effect of the angiogenic inhibitor [18].

Finally, continuous infusion therapy is applicable within clinical environment, but not yet as a portable device. However, prolonged delivery of antiangiogenic drugs may be carried out by mini-osmotic pumps or cell encapsulation systems [9, 19]. In the study [20], endostatin was microencapsulated into poly(lactic-coglycolic acid) (PLGA) microspheres to treat Lewis lung cancer. They found that in vivo releasing endostatin was biologically active and significantly inhibited the migration of endothelial cells.

Several studies examined the antitumor effect of endostatin as a function of delivery (for instance in the case of pancreatic cancer $[10,21])$. Continuous administration (using micro-osmotic pumps) was found to be more effective (97\% inhibition of tumor growth) than daily bolus doses (66\% inhibition of tumor growth), using the same dosage $(20 \mathrm{mg} / \mathrm{kg} /$ day $)$.

To sum up, there is a lot of supporting evidence from the medical experiments showing that low-dose administration of antiangiogenic drugs is more effective than high-dose delivery. Continuous drug administration may have even better efficacy; however, long-term continuous therapy raises technical questions which are not solved yet. Therefore we draw attention to the discrete-time control as an alternative and promising solution for antiangiogenic drug delivery.

\section{B. Simulation results of the protocol based cancer therapies}

We simulated the effect of the different medical protocols on the tumor growth model described by (1)-(3). The therapies were designed to be comparable; therefore the total administered inhibitor concentration was $300 \mathrm{mg} / \mathrm{kg}$ in all cases. Treatment period was 15 days in every case.

In the case of bolus doses with maximum tolerated dose (BD MTD), $100 \mathrm{mg} / \mathrm{kg}$ bolus was administered once a day in the 1st, 6th and 12th days of the treatment (3 times). The length of the drug infusion was chosen to be one hour. The rest periods were 5 days.

When bolus doses (BD) were used in lower dosage, $20 \mathrm{mg} / \mathrm{kg}$ bolus was delivered once a day every day of the treatment (15 times). The length of the drug infusion was chosen to be one hour, similarly to the BD MTD therapy. Consequently, the rest periods were 23 hours.

Using low-dose metronomic regimen (LDM), $2.5 \mathrm{mg} / \mathrm{kg}$ infusion was delivered for one day in the 1st, 4th, 7th, 10th and 13th days of the treatment (5 times). The rest periods were 2 days.

In the case of continuous infusion therapy, $0.8333 \mathrm{mg} / \mathrm{kg} / \mathrm{h}$ continuous infusion was used during the whole treatment, without rest periods.

Fig. 1 shows the results of the simulations. The less effective treatment was BD MTD, since this therapy reduced the tumor volume in the least amount (steady state tumor volume is $16330 \mathrm{~mm}^{3}$ ). This is consistent with the above mentioned clinical experimental results. In addition, maximum tolerated doses are not just therapeutically ineffective, but may cause serious adverse events for the patient, and therefore erode the quality of life.

The effectiveness of BD and LDM therapies was similar. Using daily bolus doses, the steady state tumor volume is $15580 \mathrm{~mm}^{3}$, while low-dose metronomic therapy resulted in $15660 \mathrm{~mm}^{3}$ steady state tumor volume. Despite the similar results relating to steady state tumor volume, the aspect of physiological load has to be taken into account as well. For a long-term treatment, low-dose metronomic regimen can be more tolerable, and as it can be seen from Fig. 1, due to the smaller drug dosage, vascular volume changes more slowly and to a lesser extent in LDM therapy in contrast to BD treatment.

The simulation results of continuous infusion therapy (marked as "cont" in Fig. 1) lend support to the clinical experimental results, since the lowest steady state tumor volume $\left(15360 \mathrm{~mm}^{3}\right)$ was achieved using this treatment. Other advantages of this method are the smooth change 

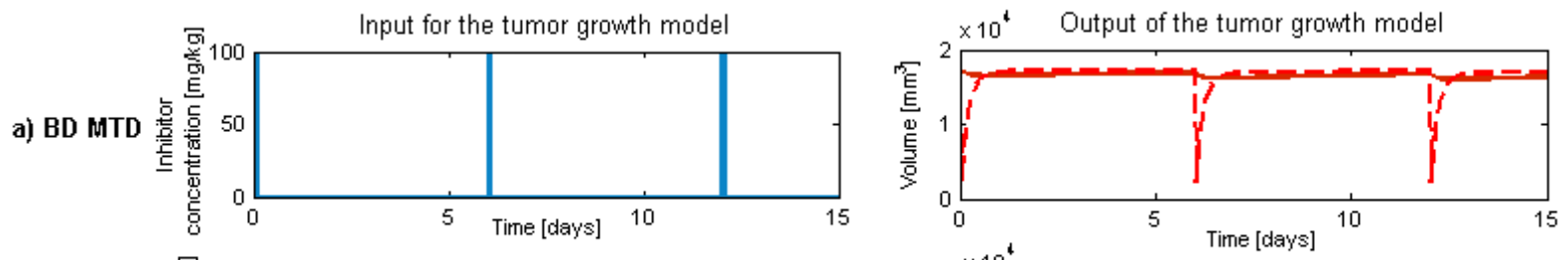

b) $B D$
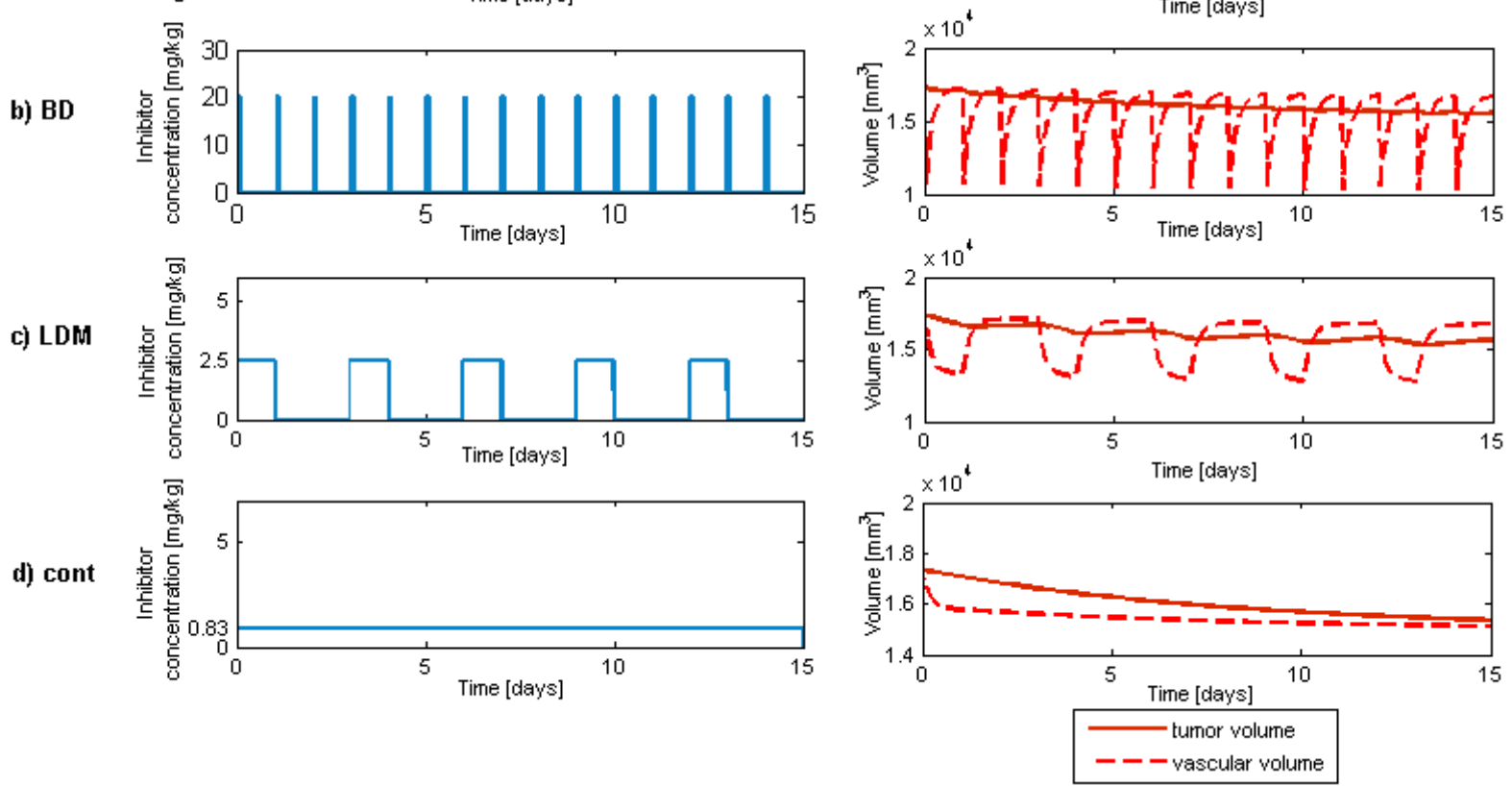

Fig. 1. Protocol based therapies. Treatment period: 15 days.

a) Bolus doses with maximum tolerated dose (BD MTD)

Therapy: $100 \mathrm{mg} / \mathrm{kg}$ bolus once a day in the $1 \mathrm{st}$, 6 th and 12 th days of the treatment; rest periods: 5 days. Total inhibitor concentration: $300 \mathrm{mg} / \mathrm{kg}$, steady state tumor volume: $16330 \mathrm{~mm}^{3}$.

\section{b) Bolus doses (BD)}

Therapy: $20 \mathrm{mg} / \mathrm{kg}$ bolus once a day every day of the treatment; rest periods: 23 hours. Total inhibitor concentration: $300 \mathrm{mg} / \mathrm{kg}$, steady state tumor volume: $15580 \mathrm{~mm}^{3}$. c) Low-dose metronomic regimen (LDM)

Therapy: $2.5 \mathrm{mg} / \mathrm{kg}$ infusion for one day in the 1st, 4th, 7th, 10th, 13th days of the treatment; rest periods: 2 days. Total inhibitor concentration: $300 \mathrm{mg} / \mathrm{kg}$, steady state tumor volume: $15660 \mathrm{~mm}^{3}$. d) Continuous infusion therapy (cont)

Therapy: $0.8333 \mathrm{mg} / \mathrm{kg} / \mathrm{h}$ continuous infusion therapy during the whole treatment; no rest periods. Total inhibitor concentration: $300 \mathrm{mg} / \mathrm{kg}$, steady state tumor volume: $15360 \mathrm{~mm}^{3}$.

of vascular volume during the treatment on the one hand, and minimal or no side effects on the other hand. However, we would like to emphasize once more that continuous long-term therapy is not yet available nowadays.

\section{DISCRETE TIME CONTROLLER BASED TREATMENTS}

\section{A. Discrete time controller with state feedback, setpoint control, actual state observer and load estimation}

Detailed description of the discrete-time controller design can be found in [7]. Here we give a concise summary of the design aspects of the discrete time controller with state feedback, setpoint control, actual state observer and load estimation.

Taking into account that state feedback control design requires linear model, we applied operating point linearization in the $g_{0}=0$ working point. The matrices of the linear model with system matrix $A$, input matrix $B$ (i.e. the right-hand side of its differential equation is $\dot{x}=A x+B u$ ), and output defined as $y=C x+D u$ are

$$
\begin{aligned}
& A=\left[\begin{array}{cc}
-\lambda_{1} \log \left(\frac{x_{1}}{x_{2}}\right)-\lambda_{1} & \lambda_{1} \frac{x_{1}}{x_{2}} \\
b-\frac{2}{3} d \cdot x_{1}^{-\frac{1}{3}} \cdot x_{2} & -d \cdot x_{1}^{\frac{2}{3}}
\end{array}\right] \\
& B=\left[\begin{array}{c}
0 \\
-e x_{2}
\end{array}\right] \\
& C=\left[\begin{array}{ll}
1 & 0
\end{array}\right] \\
& D=\left[\begin{array}{ll}
0
\end{array}\right]
\end{aligned}
$$

The whole controller structure was designed for the linearized and discretized tumor growth model; however the simulations were carried out on the original nonlinear continuous model.

Sampling time was chosen to fulfill the conditions of Shannon theorem for every signal of the accelerated (closed-loop) system. The continuous-time linear model was transformed to a discretized model that has equivalent step response in the sampling times, thus the difference equation of the discrete-time model is 


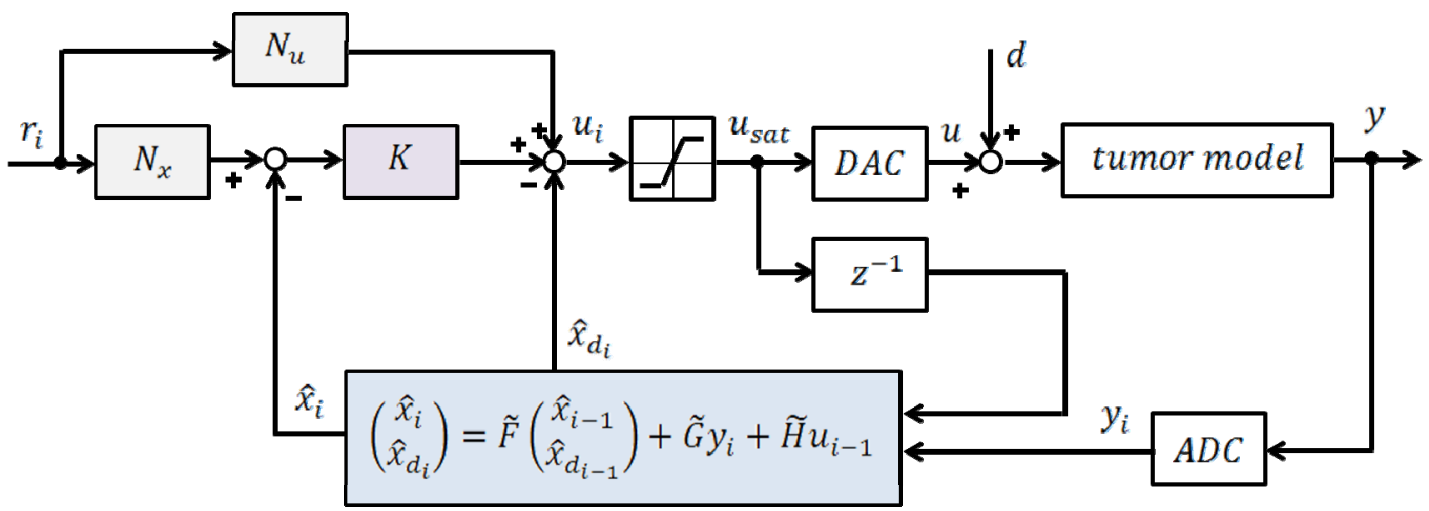

Fig. 2. Block diagram of the discrete time control containing state feedback, setpoint control, actual state observer and load estimation.

$$
x_{k+1}=A_{d} x_{k}+B_{d} u_{k} .
$$

We investigated the observability and controllability of the linearized discrete model, and we found that the matrices are full rank for every nonzero operating point, thus the system is controllable and observable.

State feedback control problem was solved by two different control methods. In the case of pole placement, the feedback matrix $K$ was determined by the Ackermann's formula, i.e.

$$
K_{P P}=e_{n}^{T} M_{C}^{-1}\left(A_{d}, B_{d}\right) \varphi_{\text {closed }}\left(A_{d}\right),
$$

where $e_{n}$ is the $n$th unit vector, $\varphi_{\text {closed }}\left(A_{d}\right)$ is the characteristic polynomial of the closed loop evaluated at the matrix $A_{d}$ and $M_{c}$ is the controllability matrix evaluated as

$$
M_{C}\left(A_{d}, B_{d}\right)=\left(\begin{array}{llll}
B_{d} & A_{d} B_{d} & \ldots & A_{d}^{n-1} B_{d}
\end{array}\right) .
$$

The feedback matrix $K$ was also calculated for the discrete time LQ problem using the formula

$$
K_{L Q}=\left(R+B_{d}{ }^{T} P B_{d}\right)^{-1} B_{d}{ }^{T} P A_{d},
$$

where $P$ is the solution of the Discrete-time Algebraic Ricatti Equation (DARE)

$$
P=A_{d}{ }^{T} P A_{d}-\left(A_{d}{ }^{T} P B_{d}\right)\left(R+B_{d}{ }^{T} P B_{d}\right)^{-1}\left(B_{d}{ }^{T} P A_{d}\right)+Q
$$

where $Q=C^{T} C$.

For setpoint control, two matrices $\left(N_{x}\right.$ and $\left.N_{u}\right)$ are needed to extend the control structure (provided that the reference signal is piecewise continuous):

$$
\left(\begin{array}{c}
N_{x} \\
N_{u}
\end{array}\right)=\left[\begin{array}{cc}
A_{d}-I & B_{d} \\
C & 0
\end{array}\right]^{-1}\left(\begin{array}{c}
0_{n x m} \\
I_{m}
\end{array}\right) .
$$

Actual state observer was designed to estimate the nonmeasurable state variables. It is described by the difference equation

where

$$
\hat{x}_{i}=F \hat{x}_{i-1}+G y_{i}+H u_{i-1},
$$

$$
\begin{aligned}
& F=A_{d}-G C A_{d} \\
& H=B_{d}-G C B_{d} \\
& G=\left(\mathrm{e}_{\mathrm{n}}^{\mathrm{T}} M_{c}^{-1}\left(A_{d}^{T}, A_{d}^{T} C_{d}^{T}\right) \varphi_{F}\left(A_{d}^{T}\right)\right)^{T},
\end{aligned}
$$

where $\varphi_{F}\left(A_{d}{ }^{T}\right)$ refers to the characteristic polynomial of the matrix $F$ evaluated at the matrix $A_{d}^{T}$.
We also designed load estimation; we assumed that the disturbance is reduced to the input of the system (load change) and it is piecewise constant, so the discrete-time model was extended by the load modeled as a constant state-variable that adds up to the input of the original model.

The state feedback and the setpoint control was designed for the original system; however, the actual state observer was designed for the extended system, whose difference equation is

$$
\left(\begin{array}{c}
\hat{x}_{i} \\
\hat{x}_{d i}
\end{array}\right)=\widetilde{F}\left(\begin{array}{c}
\hat{x}_{i-1} \\
\hat{x}_{d i-1}
\end{array}\right)+\widetilde{G} y_{i}+\widetilde{H} u_{i-1} .
$$

Fig. 2 shows the whole closed-loop control system containing the controller and the nonlinear system. We placed saturation between the tumor model and the controller; in addition, control input has a lower and an upper limit due to physiological considerations.

\section{B. Simulation results of the discrete-time controller based treatments}

Discrete controller based therapies are automatic treatments which can contain bolus doses and metronomic periods as inputs. The whole treatment periods are the same as in protocol based cancer therapies, i.e. 15 days. Each discrete controller has the following parameters (which were chosen according to [7]). The operating point, where the original nonlinear system was linearized, is $10 \mathrm{~mm}^{3}$. Controllers use LQ optimal control as a state feedback method. The value of $R$ weighting matrix is 1 . The reference signal is $13000 \mathrm{~mm}^{3}$ to get steady state tumor volume results close to the protocol based cancer therapies' values. The disturbance was chosen to be $0 \%$, since protocol based cancer therapies do not have disturbance either in the simulations. The controllers differ only in the chosen saturation level.

Fig. 3 shows the results of the simulations. Using the maximum tolerated dose as the saturation level (100 $\mathrm{mg} / \mathrm{kg}$, note that this is the same value as it was at the protocol based therapies' BD MTD case), there are more than one rest periods, and these periods are not located at equal intervals. The therapy begins with a maximum bolus dose, and after that, a half day rest period follows. The next phase takes 3 days; during this period, maximum bolus doses follow each other in about 5-10 hours. After that an approximately 6.5-day rest period follows. This 

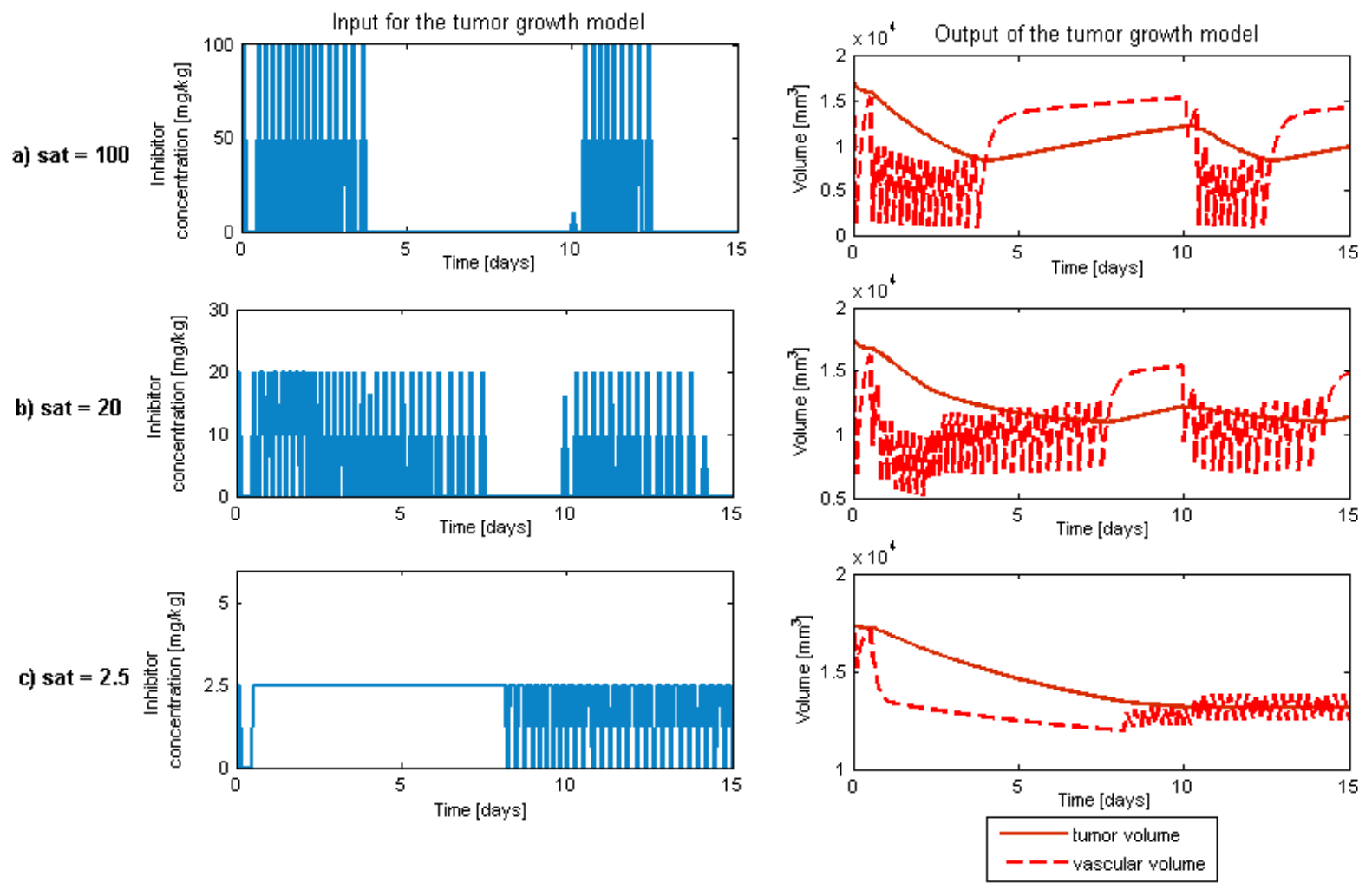

Fig. 3. Discrete controller based therapies. Treatment period: 15 days.

Parameters: operating point: $10 \mathrm{~mm}^{3} ; R: 1$; reference signal: $13000 \mathrm{~mm}^{3}$; disturbance: $0 \%$. a) Saturation: $100 \mathrm{mg} / \mathrm{kg}$

Total inhibitor concentration: $138 \mathrm{mg} / \mathrm{kg}$, steady state tumor volume: $9870 \mathrm{~mm}^{3}$.

b) Saturation: $20 \mathrm{mg} / \mathrm{kg}$

Total inhibitor concentration: $56 \mathrm{mg} / \mathrm{kg}$, steady state tumor volume: $11360 \mathrm{~mm}^{3}$.

c) Saturation: $2.5 \mathrm{mg} / \mathrm{kg}$

Total inhibitor concentration: $30 \mathrm{mg} / \mathrm{kg}$, steady state tumor volume: $13153 \mathrm{~mm}^{3}$.

ends with a $10 \mathrm{mg} / \mathrm{kg}$ bolus dose; and then a 2-day period occurs again with maximum bolus doses following each other in about 5-10 hours. The therapy ends with a 2.5 -day rest period. The total inhibitor concentration is $138 \mathrm{mg} / \mathrm{kg}$, and the achieved steady state tumor volume is $9870 \mathrm{~mm}^{3}$. As it can be seen from Fig. 3, at the end of the 15th day, the output value shows an undershoot; this is the explanation of the great difference from the setpoint.

In the second case, the saturation was chosen to be $20 \mathrm{mg} / \mathrm{kg}$ (similarly to the protocol based therapies' BD case). This treatment also has three rest periods; however, these rest periods are shorter compared to the maximum tolerated dose saturation case. In turn, the characteristics of the treatments are really similar: after the very first bolus dose, the shortest rest period occurs first, which is followed by the longest bolus dose period, and then the longest rest period appears, which is followed by a shorter bolus dose period, and finally, the second longest rest period comes. The bolus doses are not equal to the saturation value in every case (there are lower boluses). The total inhibitor concentration is $56 \mathrm{mg} / \mathrm{kg}$, and the gained steady state tumor volume is $11360 \mathrm{~mm}^{3}$. Since the input boluses (and hence the over-and undershoots) are not as high as the MTD, the steady state tumor volume approximates the setpoint better.

The third discrete controller has $2.5 \mathrm{mg} / \mathrm{kg}$ saturation limit (which is the same value as it was at the protocol based therapies' LDM case). Having regard to the low possible value of the input, to reach the setpoint, the control contains only one short rest period at the beginning of the treatment (approximately half a day). After that a 7.5-day prolonged period comes where the input keeps the value of saturation. The treatment ends with a 6.5-day long period where maximum bolus doses follow each other in about 5 hours. The total inhibitor concentration during the treatment is $30 \mathrm{mg} / \mathrm{kg}$, and the achieved steady state tumor volume is $13153 \mathrm{~mm}^{3}$.

\section{RESULTS AND DISCUSSION}

Comparison of the therapies as a function of total inhibitor concentration and steady state tumor volume can be seen in Fig. 4.

Since the protocol based therapies were designed to be comparable (the total administered inhibitor concentration was $300 \mathrm{mg} / \mathrm{kg}$ in all cases), the efficacy order can be easily set up. Bolus doses therapy with maximum tolerated dose (BD MTD) is the less effective in terms of tumor volume reduction. Bolus doses therapy with lower dose (BD) and low-dose metronomic regimen (LDM) has similar results, and continuous infusion therapy reduces the tumor volume in the greatest extent.

However, it is trivial from Fig. 4, that even the best protocol based therapy cannot approach the effectiveness of discrete controller based therapies, since these 


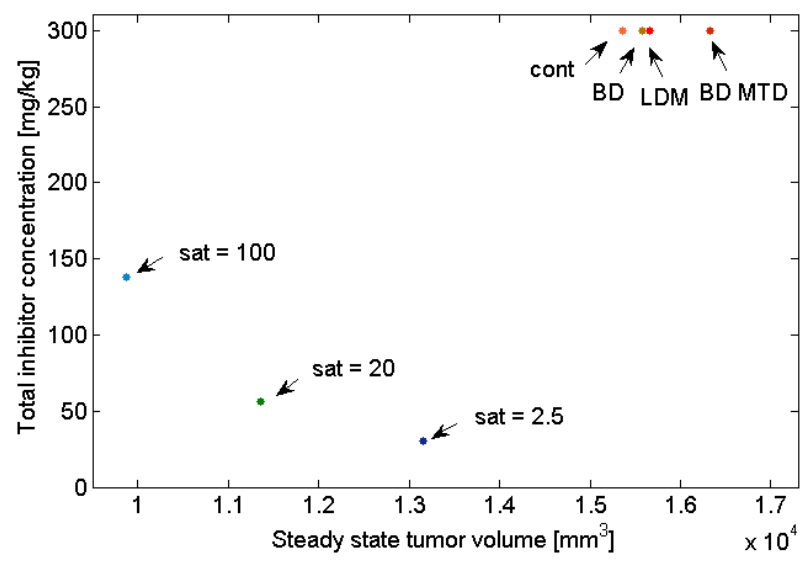

Fig. 4. Comparison of the therapies as a function of total inhibitor concentration and steady state tumor volume. Protocol based therapies: bolus doses with maximum tolerated dose (BD MTD), bolus doses (BD) low-dose metronomic regimen (LDM), continuous infusion therapy (cont). Discrete controller based therapies: saturation: $100 \mathrm{mg} / \mathrm{kg}$ (sat = $100)$, saturation: $20 \mathrm{mg} / \mathrm{kg}(\mathrm{sat}=20)$, saturation: $2.5 \mathrm{mg} / \mathrm{kg}(\mathrm{sat}=2.5)$.

automated treatments achieve considerably lower steady state tumor volumes while using less than half total inhibitor concentration. The choice between the automated treatments depends on the current therapeutic goal. If the goal is to reduce the tumor volume as fast as possible, and the patient tolerates MTD boluses, the $100 \mathrm{mg} / \mathrm{kg}$ saturation is the most appropriate way. The $20 \mathrm{mg} / \mathrm{kg}$ saturation level is a trade-off choice: the physiological load of the patient is less; however, the gained steady state tumor volume is higher compared to the BD MTD therapy. Taking into account the above mentioned vasculature normalization aspect and the minimal side effects; $2.5 \mathrm{mg} / \mathrm{kg}$ saturation seems to be the best therapeutic choice in most cases.

\section{ACKNOWLEDGEMENT}

The research was supported by the Research and Innovation Center of Obuda University and by the European Research Council Starting Grant ERC-StG 679681.

\section{REFERENCES}

[1] DE. Gerber, "Targeted therapies: a new generation of cancer treatments", Am Fam Physician, vol. 77(3), pp. 311-319, 2008.

[2] TA. Baudino, "Targeted Cancer Therapy: The Next Generation of Cancer Treatment", Curr Drug Discov Technol, vol. 12(1), pp. 320, 2015.

[3] Y. Kubota, "Tumor angiogenesis and anti-angiogenic therapy", Keio J Med., vol. 61(2), pp. 47-56, 2012.

[4] L. Amit, I. Ben-Aharon, L. Vidal, L. Leibovici, and S. Stemmer, "The impact of Bevacizumab (Avastin) on survival in metastatic solid tumors--a meta-analysis and systematic review", PLoS One, 8(1):e51780. doi: 10.1371/journal.pone.0051780. 2013.

[5] Y. Cao, and L. Xue, "Angiostatin", Semin Thromb Hemost, vol 30(1), pp. 83-93, 2004.

[6] MS. O'Reilly, T. Boehm, Y. Shing, N. Fukai, G. Vasios, WS Lane, E. Flynn, JR. Birkhead, BR. Olsen, and J. Folkman, "Endostatin: an endogenous inhibitor of angiogenesis and tumor growth", Cell, vol. 88(2), pp. 277-285, 1997.
[7] J. Sápi, DA. Drexler, and L. Kovács, "Discrete time state feedback with setpoint control, actual state observer and load estimation for a tumor growth model", submitted to SACI2016 - 11th IEEE International Symposium on Applied Computational Intelligence and Informatics, 07.03.2016

[8] DA. Drexler, J. Sápi, and L. Kovács, "On the optimal discrete time control of antiangiogenic tumor therapy", submitted to CDC2016 55th IEEE Conference on Decision and Control, 14.03.2016

[9] F. Xu, Q. Ma, and H. Sha, "Optimizing drug delivery for enhancing therapeutic efficacy of recombinant human endostatin in cancer treatment", Crit Rev Ther Drug Carrier Syst, vol. 24(5), pp. 445-492, 2007.

[10] O. Kisker, CM. Becker, D. Prox, M. Fannon, R. D'Amato, E. Flynn, WE. Fogler, BK. Sim, EN. Allred, SR. Pirie-Shepherd, and J. Folkman, "Continuous administration of endostatin by intraperitoneally implanted osmotic pump improves the efficacy and potency of therapy in a mouse xenograft tumor model", Cancer Res, vol. 61(20), pp. 7669-7674, 2001.

[11] P. Hahnfeldt, D. Panigrahy, J. Folkman, and L. Hlatky, "Tumor development under angiogenic signaling: A dynamical theory of tumor growth, treatment response, and postvascular dormancy", Cancer research, vol. 59, pp. 4770-4775, 1999.

[12] U. Ledzewicz, and H. Schätler, "A synthesis of optimal controls for a model of tumor growth under angiogenic inhibitors", $C D C$ 2005 - 44th IEEE Conference on Decision and Control, and the European Control Conference, pp. 934-939, December 2005, Sevilla, Spain.

[13] O.G. Scharovsky, L.E. Mainetti, and V.R. Rozados. Metronomic chemotherapy: changing the paradigm that more is better. Current Oncology, 16(2):7-15, 2009.

[14] T. Browder, C. E. Butterfield, B. M. Kr"aling, B Shi, B. Marshall, M. S. O'Reilly, and J. Folkman. Antiangiogenic scheduling of chemotherapy improves efficacy against experimental drugresistant cancer. Cancer Research, 60(7):1878-1886, 2000

[15] R. S. Kerbel. Improving conventional or low dose metronomic chemotherapy with targeted antiangiogenic drugs. Cancer Research and Treatment, 39(4):150-159, 2007.

[16] J. Sápi, L. Kovács, D.A. Drexler, P. Kocsis, D. Gajári, and Z. Sápi, "Tumor Volume Estimation and Quasi-Continuous Administration for Most Effective Bevacizumab Therapy", PloS One, vol. 10:(11), Paper e142190. 20 p, 2015.

[17] T. Ning, M. Jiang, Q. Peng, X. Yan, Z.-J. Lu, Y.-L.Peng, et al., "Low-dose endostatin normalizes the structure and function of tumor vasculature and improves the delivery and anti-tumor efficacy of cytotoxic drugs in a lung cancer xenograft murine model", Thorac. Cancer, vol. 3(2012), pp. 229-238, 2012.

[18] RK. Jain, "Normalization of tumor vasculature: an emerging concept in antiangiogenic therapy", Science, vol. 307(5706), pp. $58-62,2005$.

[19] DR. Sorensen, TA. Read, "Delivery of endostatin in experimental cancer therapy", Int J Exp Pathol, vol. 83(6), pp. 265-274, 2002.

[20] J. Wu, D. Ding, G. Ren, X. Xu, X. Yin, Y. Hu, "Sustained delivery of endostatin improves the efficacy of therapy in Lewis lung cancer model", J Control Release, vol. 134(2), pp. 91-7, 2009 .

[21] M. Capillo, P. Mancuso, A. Gobbi, S. Monestiroli, G. Pruneri, C. Dell'Agnola, G. Martinelli, L. Shultz, F. Bertolini, "Continuous infusion of endostatin inhibits differentiation, mobilization, and clonogenic potential of endothelial cell progenitors", Clin Cancer Res. vol. 9(1), pp. 377-382, 2003. 